\title{
Mucosal Lentiginous Melanoma
}

National Cancer Institute

\section{Source}

National Cancer Institute. Mucosal Lentiginous Melanoma. NCI Thesaurus. Code C48622.

An acral lentiginous melanoma affecting mucosal surfaces. 